\title{
REVIEW
}

\section{Genetic regulatory pathways of split-hand/foot malformation}

\author{
Piranit N. Kantaputra ${ }^{1,2,3}$ @ | Bruce M. Carlson ${ }^{4}$
}

${ }^{1}$ Center of Excellence in Medical Genetics Research, Chiang Mai University, Chiang Mai, Thailand

${ }^{2}$ Division of Pediatric Dentistry, Department of Orthodontics and Pediatric Dentistry,

Faculty of Dentistry, Chiang Mai University, Chiang Mai, Thailand

${ }^{3}$ Dentaland Clinic, Chiang Mai, Thailand

${ }^{4}$ Department of Anatomy and Cell Biology, University of Michigan, Ann Arbor, Michigan

\section{Correspondence}

Piranit N. Kantaputra, Division of Pediatric Dentistry, Department of Orthodontics and Pediatric Dentistry, Faculty of Dentistry, Chiang Mai University, Dentaland Clinic, Chiang Mai 50200, Thailand.

Email: dentaland17@gmail.com

Funding information

Center of Excellence in Medical Genetic Research, Chiang Mai University, Grant/Award Number: N/A; Dental Association of Thailand, Grant/Award Number: N/A; Faculty of Dentistry, Chiang Mai University, Grant/Award Number: N/A; Thailand Research Fund, Grant/ Award Number: BRG 6180007

The peer review history for this article is available at https://publons.com/publon/ 10.1111/cge.13434/

\begin{abstract}
Split-hand/foot malformation (SHFM) is caused by mutations in TP63, DLX5, DLX6, FGF8, FGFR1, WNT10B, and BHLHA9. The clinical features of SHFM caused by mutations of these genes are not distinguishable. This implies that in normal situations these SHFM-associated genes share an underlying regulatory pathway that is involved in the development of the central parts of the hands and feet. The mutations in SHFM-related genes lead to dysregulation of Fgf8 in the central portion of the apical ectodermal ridge (AER) and subsequently lead to misexpression of a number of downstream target genes, failure of stratification of the AER, and thus SHFM. Syndactyly of the remaining digits is most likely the effects of dysregulation of Fgf-BmpMsx signaling on apoptotic cell death. Loss of digit identity in SHFM is hypothesized to be the effects of misexpression of HOX genes, abnormal SHH gradient, or the loss of balance between GLI3A and GLI3R. Disruption of canonical and non-canonical Wnt signaling is involved in the pathogenesis of SHFM. Whatever the causative genes of SHFM are, the mutations seem to lead to dysregulation of Fgf8 in AER cells of the central parts of the hands and feet and disruption of Wnt-Bmp-Fgf signaling pathways in AER.
\end{abstract}

\section{KEYWORDS}

ectrodactyly, lobster claw deformity, malformation of limb, oligodactyly, SHFM

\section{1 | INTRODUCTION}

Split-hand/foot malformation (SHFM) is a congenital limb malformation predominantly affecting the central rays of the hands and/or feet (MIM \#183600, \#313350, \#600095, \#605289, and \#606708). The clinical features of SHFM are highly variable and asymmetrical. ${ }^{1}$ They range from mild defects such as a hypoplasia of a single phalanx or syndactyly, to aplasia of one or more central digits. Intra-familial and inter-individual variability of the SHFM are very high (Figure 1). Phenotypic variability is most likely the result of genetic background including modifier genes, epigenetic and environmental factors. The condition is clinically and genetically heterogeneous. It may be isolated (non-syndromic) or syndromic. Most cases show autosomal dominant inheritance with variable expressivity and reduced penetrance. Pathogenesis of SHFM is directly and indirectly related to maldevelopment of the apical ectodermal ridge (AER), a specialized stratified columnar epithelium locating at the distal rim of the developing limb bud. The AER is the transitory major signaling center for proximodistal growth and distal limb development. ${ }^{2}$ It keeps the underlying mesenchyme in a proliferative and undifferentiated stage, preventing cell death, and subsequently allowing the developing limb to elongate. The AER is induced through the reciprocal interactions between the ectoderm and the underlying mesenchyme involving Wnt-Bmp-Fgf signaling pathways. ${ }^{3,4}$ Disruption of these pathways in the AER generally lead to SHFM.

\section{2 | SHFM-ASSOCIATED SYNDROMES}

SHFM has been reported to be associated with ectrodactyly-ectodermal dysplasia-clefting syndrome (EEC; MIM \#129900), ${ }^{5}$ brachydactylyectrodactyly with fibular aplasia or hypoplasia (MIM \#113310), ${ }^{6}$ autosomal recessive DLX5-associated $\mathrm{SHFM}^{7}$ autosomal dominant DLX5associated SHFM, ${ }^{8}$ autosomal dominant DLX6-associated SHFM, ${ }^{9}$ 


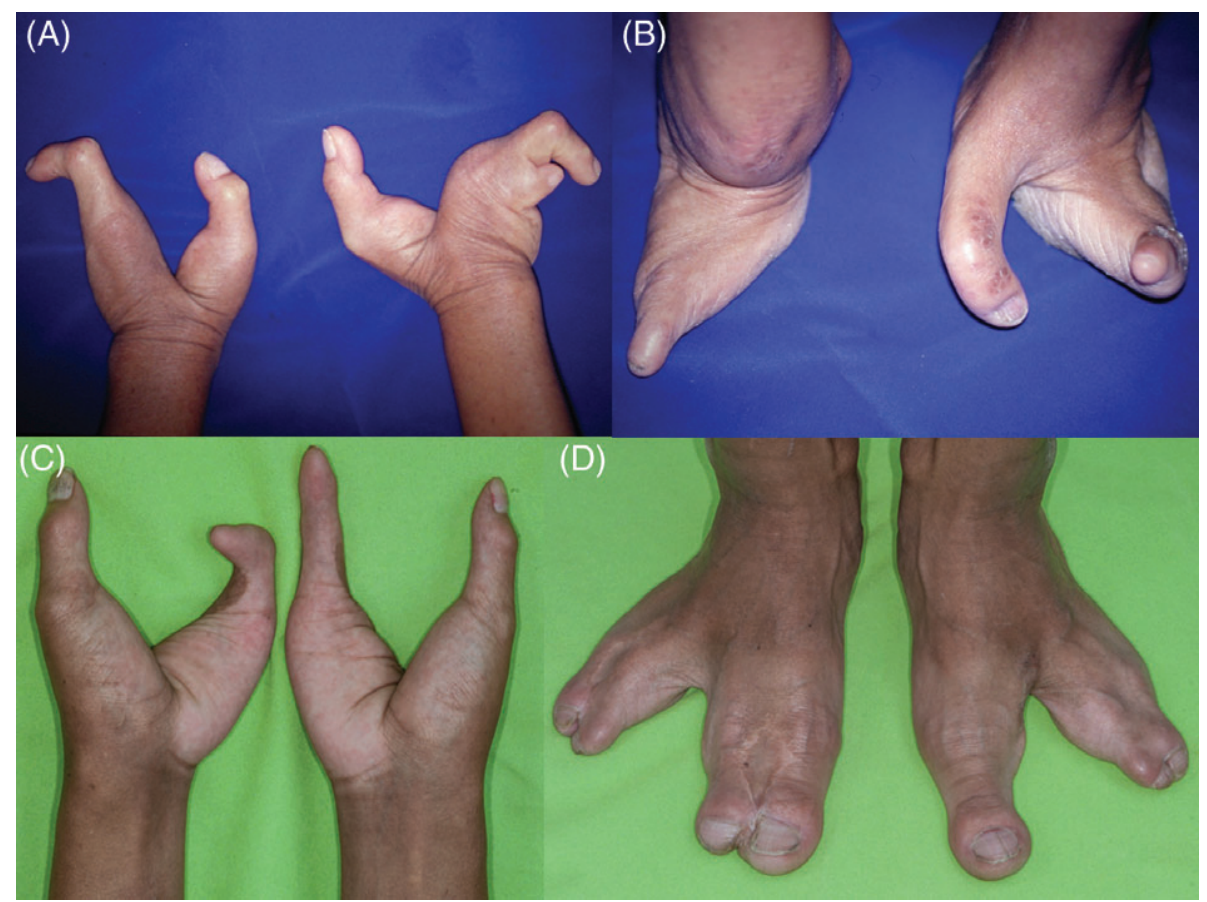

FIGURE 1 Split-hand/foot malformation caused by a mutation in TP63. A and B, Mother. C and D, Son. Note loss of mirror-image morphogenesis or asymmetric manifestaitons of hands and feet. Note loss of phynotypic identity of digits

FGFR1-associated congenital hypogonadotropic hypogonadism with SHFM (MIM \#147950), ${ }^{10}$ BHLHA9-associated SHFM with long-bone deficiency (SHFMLD: MIM \#119100), ${ }^{11,12}$ and autosomal recessive WNT10B-associated SHFM (SHFM6; MIM \#225300). ${ }^{1}$ A patient with Kabuki syndrome with SHFM has been reported, but molecular testing was not performed. ${ }^{13}$ Being associated with a number of syndromes implies that pathogenesis of SHFM involves a number of genes and these genes are likely to share the pathogenetic pathways involving in developing the central part of the hands and feet. It is important to note that "atypical" SHFM can be seen in patients affected with PORCN-associated Goltz-Gorlin or cohesin complex-associated Cornelia-de Lange syndrome, but here we focus only on the WNT-TP63-DLX-associated SHFM.

\section{3 | SHFM-ASSOCIATED GENES}

Mutations in TP63, DLX5, DLX6, FGFR1, BHLHA9, and WNT10B are known to be associated with SHFM in humans, and interestingly the clinical features of SHFM caused by mutations of these genes are not distinguishable. ${ }^{9,14,15}$ This implies that in a normal situation these SHFM-associated genes share in part an underlying regulatory pathway that is involved in development of hands and feet (Figure 2). These genes are found to be co-expressed in the AER cells of the developing mouse limb $^{2}$ and the developing fin of zebrafish. ${ }^{16}$ Interestingly, SHFM has also been observed in mice, amphibians, and chickens, indicating the similar and conserved genetic signaling pathways associated with SHFM in various species. ${ }^{4}$

\section{1 | TP63 and SHFM}

p63 (MIM \#603273), a homolog to p53 and p73, codes for transcription factor p63. p63 is expressed in ectoderm-derived tissues. Its role is to initiate epithelial stratification program during early embryonic development. Mutations in TP63 have been reported to be associated with various kinds of human malformations including SHFMassociated EEC syndrome and isolated SHFM. ${ }^{5}$ p63 has crucial roles in forming AER and controlling AER functions via transcriptional regulation of AER-restricted target genes including Dlx5, Fgf8, Sp6, Sp8, and Msx1 (Figure 2). The $\Delta \mathrm{Np} 63 \alpha$ isoform, the most expressed isoform of embryonic ectoderm, ${ }^{14}$ is the predominant p63 isoform expressed in developing limb. It is the main regulatory isoform that has been shown to induce transcription of the Dlx5 and Dlx6 promoters in vitro (Figure 2). ${ }^{2}$ $p 63^{-/-}$mice have abnormal expression of AER-restricted target genes, failure of AER formation, and subsequent absent hindlimbs and severely truncated forelimbs (Figure 2). ${ }^{3,17,18}$ Failure of AER stratification subsequent to disruption of p63-Dlx signaling pathway might be the result of dysregulation of cell adhesion-associated $p 63$ target genes including Perp and $\mathrm{CDH} 3$. Both genes have important roles in cell adhesion, and maintenance of epithelial integrity and mutations in $\mathrm{CDH} 3$ have been shown to cause SHFM in autosomal recessive ectodermal dysplasiaectrodactyly-macular dystrophy syndrome (EEMS; MIM \#225280). ${ }^{19}$ It is noteworthy that limb defects related to p63 mutations or p63-associated pathways appear to be more severe on the hindlimbs than the forelimbs. However, only the TP63 mutations that cause EEC syndrome and isolated SHFM but not ankyloblepharon-ectodermal defects-cleft lip/palate (AEC; MIM \#106206) syndrome lose the ability to induce the transcription of the DIx5 and Dlx6 by cis-acting regulation at the promoter level in vitro. ${ }^{2}$ This implies that the SHFM caused by TP63 mutations depends on the exact nature of the mutations.

\subsection{Dlx genes and SHFM}

Dlx genes code homeodomain-containing transcription factors that are vertebrate homologs of Drosophila distalless (dII), a gene necessary for specification of the distal structures of appendages including legs. 


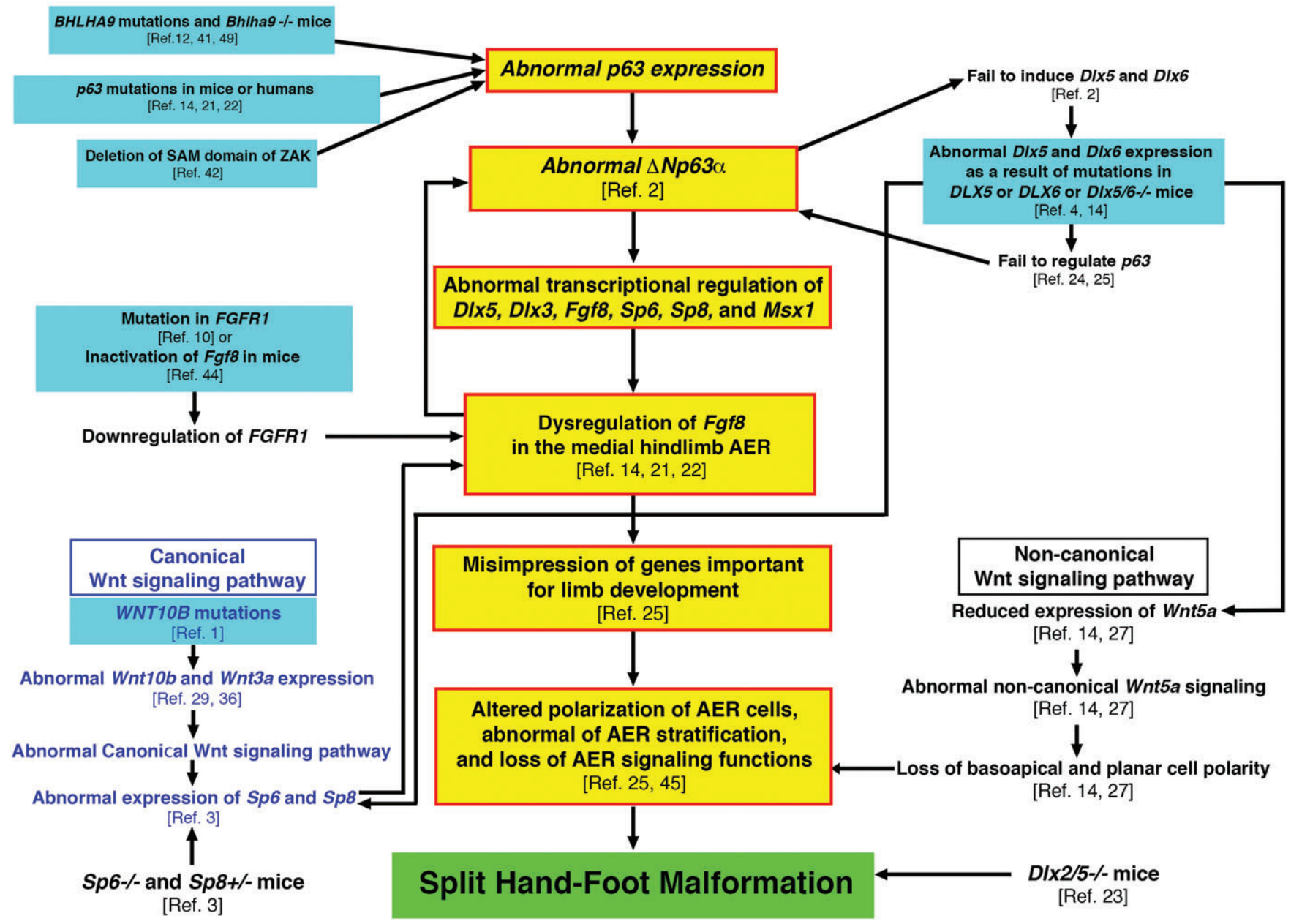

FIGURE 2 Flowchart shows genetic pathways involving split-hand/foot malformation (SHFM). Mutations that cause SHFM in mice and humans. Disruption of p63-Dlx-Fgf8 transcriptional network leads to dysregulation of Fgf8 in apical ectodermal ridge (AER) cells, misexpression of genes important for limb development, failure of AER stratification, and subsequently cause SHFM. Canonical and non-canonical Wnt signaling pathways are involved in the pathogenesis of SHFM

Mutations in DLX5 (MIM \#600028) have been reported to be associated with autosomal recessive SHFM $^{7}$ and autosomal dominant SHFM. ${ }^{8} \mathrm{~A}$ heterozygous missense mutation in DLX6 (MIM \#600030) has been reported to be associated with SHFM (Figure 2). ${ }^{20}$ Dlx5 and Dlx6 genes, are known to regulate the development of the central portion of AER during early limb development. Both genes are essential for the maintenance of the central portion of the murine hindlimb AER. In the $D / x 5 / 6^{-1-}$ mouse embryos, the expression of Fgf8 and Dlx2 is downregulated specifically in the central portion of AER of the hindlimbs, the part that would have governed the development of the central rays of the feet (Figure 2). ${ }^{14,21,22}$ This is supported by the SHFM observed in Dl $2 / 5^{-/-}$mice (Figure 2). ${ }^{23}$ The split hindlimbs in Dlx5/6 $6^{-/-}$mice are the result of a cell-autonomous failure of the central AER to regulate $p 63$ and to maintain and express morphogenetic signals (Figure 2). ${ }^{24,25}$ The degeneration of the AER in DIx5/6 $6^{-/-}$mice led to SHFM phenotype which is similar to the phenotype of Dactylaplasia mice. ${ }^{26}$ The polarization of the ectodermal cells of AER of the developing limb is regulated by non-canonical Wnt5a signaling. Dlx-related SHFM is associated with the loss of basoapical and planar cell polarity (PCP) and abnormal noncanonical Wnt5a signaling, as a result of reduced expression of Wnt5a, a transcriptional target of Dlx5 (Figure 2). ${ }^{14,27}$
Dlx5 and Dlx6 proteins are direct targets of $\Delta \mathrm{Np} 63 \alpha^{2}{ }^{2}$ p63 mutations or combined loss of Dlx5 and Dlx6 lead to downregulation of FGF8 and DIx2 expression in the central portion of AER, misexpression of genes important for limb development, and subsequent SHFM (Figure 2). 2,21,22 Dlx5 and Dlx6, and to a lesser degree Dlx1 and Dlx2, are downregulated in heterozygous $p 63^{-/+}$and $p 63^{\mathrm{EEC}}$ in the mouse hindlimbs, which have normal AER. ${ }^{2}$ Like $\mathrm{p} 63^{-/-}$mice, the hindlimbs of $D / x 5 / 6^{-1-}$ mice are more severely affected than the forelimbs. ${ }^{2,17,18}$ The $p 63^{E E C}$ mutation has different effects on forelimbs and hindlimbs, because $D / x$ genes expression is reduced in the hindlimbs but increased in the forelimbs of the $p 63$ heterozygous mice. ${ }^{2}$ SHFM in patients with TP63, DLX5, and DLX6 mutations may be the result of failure of central AER formation and maintenance, because DIx5 and Dlx6 regulate the expression of the key AER controlling gene Fgf8 in the central part of AER and p63 works upstream as their transcription factor (Figure 2).

\section{3 | WNT10B, WNT signaling, and SHFM}

Initially, limb buds form as the result of the interaction between Fgf and Wnt signaling. ${ }^{28}$ Subsequently, Wnt- $\beta$-catenin signaling has important roles in proximodistal outgrowth and dorsoventral 
patterning of the limb. Removal of $\beta$-catenin from the limb ectoderm prior to the initiation of Fgf8 expression completely prevents limb development, while its removal after Fgf8 expression results in limb truncations. ${ }^{29}$ Wnt8c, a mediator of hindlimb bud initiation, is expressed in the presumptive hindlimb region and mediates the Fgf8-Fgf10 regulatory loop via canonical Wnt- $\beta$-catenin signaling. ${ }^{30}$

The association of WNT10B (MIM \#601906) mutations and SHFM support the important roles of WNT10B and canonical Wnt$\beta$-catenin signaling pathway in the development of the central parts of hands and feet (Figure 2). ${ }^{1}$ WNT10B mutations have also been reported to be associated with isolated dental anomalies including hypodontia, microdontia, and taurodontism, ${ }^{31}$ suggesting that the phenotypes of WNT10B and TP63 mutations evidently depend on the exact nature of the mutations. Generally, Wnt10b is expressed throughout the limb bud ectoderm in all stages of limb development. However, the expression in AER is only at E11.5. ${ }^{32}$ Canonical and non-canonical Wnt signaling pathways have been known to be involved in several developmental processes at many stages of limb development. $^{28,33}$ Both pathways are required for AER formation and maintenance. Abnormal canonical Wnt- $\beta$-catenin signaling results in premature AER degeneration (Figure 2). Wnt5A is expressed in the AER and the underlying mesenchyme at the same time as p63, Dlx5, and Dlx6. Loss of the non-canonical Wnt receptors Ryx and Ror2 causes abnormal limbs similar to those of Wnt5a mutants suggesting the involvement of Wnt5a in non-canonical Wnt signaling. ${ }^{34} \mathrm{Wnt} 5 a$ via non-canonical Wnt/PCP signaling is necessary for driving proper limb morphogenesis by regulating cell organization and orienting cellular processes including mitosis and directional cell movements. ${ }^{28,33}$ Inactivation of Wnt5a results in reduced proliferation of mesenchymal cells in the progress zone and subsequent distal limb truncation. ${ }^{35}$ Evidently, pathogenesis of SHFM involves both canonical and noncanonical Wnt signaling (Figure 2).

Canonical Wnt signaling involves Wnt10b and Wnt $3 a$, and acts upstream of FGFs in establishing AER gene expression. ${ }^{29,36}$ Wnt3a acts upstream of Fgf8 during chick limb bud outgrowth and AER formation through Wnt3a/ $\beta$-catenin/Lef1 signaling. ${ }^{36}$ Different Wnt genes are able to substitute for one another, when they activate the same intracellular signaling pathways mediated by $\beta$-catenin/Lef1 signaling. ${ }^{36}$ The malformed digits in patients with SHFM might also be the result of abnormal Shh expression which is controlled by the combinatorial influence of Wnt and Fgf signaling. ${ }^{28}$

\subsection{Sp6 and Sp8 and SHFM}

Sp6 (Epiprofin; MIM \#608613) and Sp8 (Buttonhead; MIM \#608306) are members of the $\mathrm{Sp}$ zinc finger transcription factor family that have important roles in AER induction and maintenance and dorsoventral patterning of the limb. ${ }^{3}$ Both genes are expressed in the entire prospective limb ectoderm but subsequently confined to the AER during limb bud emergence. Sp6 and Sp8 share similar patterns of gene expression in limb, bud ectoderm and AER and have redundant function downstream of Wnt- $\beta$-catenin signaling in the induction of Fgf8 (Figure 2) and Bmp signaling in the induction of En1, coordinating the link between proximal-distal and dorsal-ventral patterning. ${ }^{3} \mathrm{Sp6}^{-/-}$ mice had mild syndactyly, ${ }^{37}$ while $S p 8^{-/-}$mice displayed severe limb truncation. ${ }^{38}$ Sp6 and Sp8 work together in a dose-dependent manner and are indispendable mediators of Wnt- $\beta$-catenin and Bmp signaling in developing limb ectoderm. ${ }^{3,39}$ Abnormal Wnt- $\beta$-catenin ${ }^{30}$ and Bmp signaling ${ }^{39}$ in AER have been reported to cause dysregulation of FGF signaling and subsequent SHFM. ${ }^{4} \mathrm{Sp}^{-/-} ; \mathrm{Sp} 8^{+/-}$mice had SHFM with hindlimbs more severely affected than the forelimbs (Figure 2). The digits of forelimbs and hindlimbs showed dorsalization of the digit tips. Sp6 and Sp8 work together as necessary mediators of the Wnt$\beta$-catenin-Fgf8 regulatory loop (Figure 2). The observed phenotypes of $S p 6^{-/-} ; \mathrm{Sp}^{+/-}$mice indicate that the product obtained from one allele of $S p 8$ in the absence of Sp6 is not enough for proper Fgf8 induction (Figure 2). Complete absence of Sp6 and Sp8 transcription factors does not prevent the initiation of AER morphology indicating the independence of AER function and morphology. ${ }^{3}$ Tp63, DIx5, and Dlx6 have normal expression in the early $S p 6^{-/-}, S p 8^{-/-}$limb bud implying that Sp6 and Sp8 act downstream of Tp63 and DIx5 and Dlx6. It is suggested that Wnt- $\beta$-catenin signaling is upstream of Tp63-Dlx and the Sp-Fgf8 regulatory modules (Figure 2). ${ }^{3}$

\section{5 | BHLHA9 and SHFM}

Mutations in BHLHA9 (Fingerin; MIM \#615416) have been associated with SHFMLD (Figure 2). ${ }^{12}$ BHLHA9, a member of the basic helixloop-helix $(b H L H)$ transcription factor family, has an important role in development of limb. At an early embryonic stage (E10.5), Bhlha9 is prominently expressed on the dorsal and ventral surfaces covering the progress zone near AER. It transiently regulates AER formation in the progress zone of developing limb by regulating AER formation-related genes, including Trp63 and Fgf8 (Figure 2). Bhlha9-knockout mice have various degree of syndactyly and poliosis of the limb, ${ }^{40}$ and Bhlha9knockdown zebrafish has severely truncated pectoral fins. ${ }^{11}$ Bhlha9knockout AER has overexpression and dysregulation of Trp63 and Fgf8. ${ }^{41}$ Mutations in the DNA-binding domain of BHLHA9 destroys the ability to fine tune control of regulatory pathways of limb development. $^{12}$ Abnormal expression of BHLHA9 causes SHFM and SHFMLD via dysregulation of AER formation and AER-associated genes (Figure 2).

\subsection{ZAK and SHFM}

A syndrome of split foot without split-hand malformation with mesoaxial polydactyly (MIM \#616890) has been reported to be caused by mutations in ZAK (Leucine-zipper and sterile alpha motifcontaining kinase; MIM \#609479) (Figure 2). ZAK has an important role in limb development. ZAK is a direct target of Tp63, and the deletion of its SAM domain has been shown to be associated with downregulation of $T p 63$ in the developing limb bud (Figure 2). ${ }^{42}$ The absence of split hands but the presence split feet in patients with ZAK mutations supports the control of ZAK by TP63 genes because the hindlimbs of the Tp63 KO mice are more severely affected than the forelimbs. ${ }^{17,18}$ In addition, having split feet without split hands suggest the association of ZAK, PITX1, and TBX4 genes in the formation of feet. The specification of limb identity and morphology is established prior to limb initiation. The transcription factor Tbx4 and its upstream 
regulator Pitx1 are known to have important role in defining the hindlimbs in mice, legs of chicks, and pelvic fins of fishes. ${ }^{14,43}$

\section{7 | Fgf8, FGF signaling and SHFM}

FGF signaling is known to be important in driving cell proliferation, cell survival, and specification of limb mesenchymal cells. It also promotes the velocity of cell movements within the developing limb bud, thereby promoting limb elongation. ${ }^{33}$ FGF8 is a potent ligand of FGFR1, and mutations in FGFR1 are associated with congenital hypogonadotropic hypogonadism with SHFM (Figure 2). ${ }^{10}$ Fgf8, a direct target of P63, is a key morphogen for limb outgrowth and patterning. ${ }^{17}$ It is the first AER marker during chick limb development. ${ }^{36}$ Fgf8 is initially expressed in a broad area in the distal limb ectoderm and subsequently becomes restricted to the AER. ${ }^{33}$ Fgf8 signaling is essential for AER induction and maintenance. ${ }^{44}$ Fgf8 functions are to sustain epithelial-mesenchymal signaling and assure the timely generation of the correct population of mesenchymal progenitors. ${ }^{45}$ It in turn regulates the function and stability of $\Delta \mathrm{Np} 63 \alpha$ by increasing the binding of $\Delta \mathrm{Np} 63 \alpha$ to the tyrosine kinase c-Abl and the level of $\Delta$ Np63 $\alpha$ acetylation. ${ }^{25}$ Aberration of Fgf8 expression is hypothesized to cause malfunction of $\Delta \mathrm{Np} 63 \alpha$ (Figure 2). In order for a normal limb to develop, Fgf8 is crucial for the correct establishment of the signaling loop within the developing limb bud. ${ }^{46}$ Alterations in Fgf8 expression and signaling have been shown to lead to altered reduced layer or altered adhesion in AER and subsequent limb malformations in mice. ${ }^{44}$ Reduced expression of Fgf8 and its signaling is generally linked to abnormal AER morphology and subsequent SHFM (Figure 2). However, increase of its expression in AER in lkk $\alpha$ mutant mice is associated with distal limb truncations, and overexpression of Fgf8 has been shown in Bhlha9-knockout AER. ${ }^{41}$ It is noteworthy that AER-specific Fgf8 $^{-1-}$ mice have a normal AER, suggesting that abnormal Fgf8 expression alone does not lead to SHFM. In order for mice to have SHFM, alterations in Fgf8 expression need to accompany abnormal expression of other genes in AER as well. Loss of Fgf8 or mutations in Fgf8 leads to abnormal $\Delta \mathrm{Np} 63 \alpha$ protein stability, ${ }^{24,25,44}$ failure of AER stratification, and subsequent aberrant limb development (Figure 2). ${ }^{45}$

All lines of evidence suggest that abnormalities of TP63, WNT10B, DLX5, DLX6, FGF8, and FGFR1 lead to dysregulation of FGF8 in the central portion of the AER and subsequently lead to misexpression of a number of AER genes, failure of its stratification, and thus SHFM (Figure 2).

\section{8 | Embryological mechanisms of digital patterning in SHFM}

The anatomical defect in DIx5, Dlx6-/- SHFM in the mouse has been shown to relate to a partial breakdown of the AER during the paddle stage of limb morphogenesis. ${ }^{14} \mathrm{~A}$ typical phenotype in SHFM consists of a loss of one or more central digits, with the first and fifth digits typically remaining, but often malformed. Syndactyly of the remaining digits is common and may be related to the inability of the Fgf8-related disrupted AER to regulate the expression of BMPs, Msx1, and Msx2 in the interdigital spaces. These molecules play important roles in stimulating normal interdigital cell death. Reduced expression of Msx1 and Msx2 in developing autopod leads to premature differentiation of interdigital mesoderm into connective tissue, failure of apoptotic cell death of the interdigital mesoderm, and subsequent syndactyly of the remaining digits (Figure 3). ${ }^{47,48}$

Recent reports have suggested that the putative transcription factor BHLHA9 is involved in SHFM. ${ }^{41,49}$ Both the clinical report ${ }^{49}$ and the study of Bhlha9 knockout mice ${ }^{41}$ emphasized the connection between lack of function of this gene and the presence of syndactyly that may or may not be associated with the missing or defective skeletal elements seen in SHFM. The knockout study showed that expression of Bhlha9 occurs during the period when Conte et al ${ }^{14}$ showed a reduction of Fgf8 expression in the central AER in the developing mouse limb, but the phenotypic effect appears to involve survival of interdigital soft tissue due to a diminution of apoptosis.

Because of the malformation, it is not always possible to identify the remaining digits by digit number. Digit identity, the characteristic that allows one to determine specific digits 1 to 5 , can be either embryologic or phenotypic. Embryologic identity would refer to the number of a specified digit, whereas phenotypic identity is based on clinical examination. Unfortunately, there are presently no specific markers for mammalian digits 2 to 5 that would allow one to determine their embryological identity.

Two fundamentally different processes are involved in digit formation. One is patterning (specification), which occurs in the early outgrowing limb bud. Actual formation of digital primordia occurs later, under the influence of the AER and a putative digital organizing center (see below). Digit specification means that the fate of mesenchymal cells in the hand or foot plate to develop into cartilaginous digital elements becomes fixed. By E11.5 in the mouse, when Conte et $\mathrm{al}^{14}$ identified breakdown of the AER, all five digits in the mouse limb have already been specified, ${ }^{50}$ and primordia of digits 2 to 5 are already identifiable. ${ }^{51}$

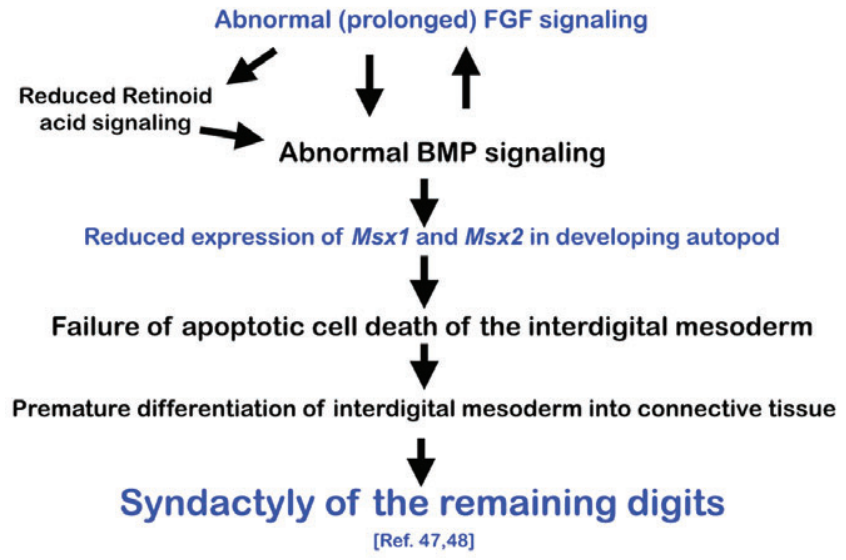

FIGURE 3 Flowchart shows genetic pathways involving syndactyly of the remaining digits in split-hand/foot malformation (SHFM). Syndactyly of the remaining digits in SHFM involves abnormal FgfBmp-retinoic acid signaling, reduced expression of Msx genes, premature differentiation of interdigital mesoderm into connective tissue, failure of apoptotic cell death, and thus syndactyly of the remaining digits 
Early patterning of the limb and the digits is based on the secretion of the morphogen Shh from the zone of polarizing activity (ZPA), located on the posterior edge of the limb bud. ${ }^{4}$ Patterning of the digits in a pentadactyl limb is largely based on the reactions of mesenchymal cells to various degrees of exposure to Shh. Digit 1 is generally conceded to be independent of an Shh influence, and it will form in the absence of a ZPA. Digits 2 to 5 are patterned on the basis of both time of exposure and concentration of mesenchymal cells to Shh. A low concentration of Shh specifies digit 2, whereas exposure to a higher concentration of Shh over a longer time specifies digit 3. Digits 4 and 5 are specified on the basis of length of exposure to high concentrations of Shh. ${ }^{50,52}$ Loss of digit identity in SHFM has been hypothesized to be the effects of misexpression of HOX genes, abnormal SHH gradient, or the loss of balance between GLI3A and GLI3R as a result of WNT10B-associated abnormal FGF signaling. ${ }^{53,54}$

Individual digits begin to take shape through the appearance of pre-chondrogenic organizing centers that form the distal aspect of outgrowing digital rays. ${ }^{55}$ Outgrowth of digits requires the action of a functional AER over the tips of the digits. While digits are taking shape, the AER over the future interdigital spaces becomes attenuated. In normal animals, mesenchymal cells accumulate, either by movements ${ }^{33}$ and/or cell division, beneath the non-attenuated segments of the AER. Given a sufficient number of mesenchymal cells, digital primordia begin to form. In the absence of sufficient cells, chondrogenesis in either limb or digital primordia fails to occur.

Based on the information available, the pathology commonly seen in the various types of SHFM suggests that the malformation does not affect the specification of the central digits because specification occurs very early, well before the earliest reported demonstration of pathology to the AER. Rather, it appears to be due in large part to the inability of the disrupted AER in mutants to support the outgrowth of the digital primordia in the central part of the hand or foot plate. It would be necessary to examine limbs at stages earlier than E11.5, as was performed by Conte et $\mathrm{al}^{14}$, in order to determine whether or not the genetic defect affects digital specification. If affected hands or feet possess a recognizable nondigit 1 digit on the anterior side, it would suggest that full initial digital specification had, indeed, occurred prior to the development of the defect because digital specification occurs in a posterior-toanterior direction, and it would be unlikely that a digit 2 could form in the absence of a specified digit 3 or 4 . If only a digit 1 is present on the anterior side, it would not be possible to comment on possible specification issues.

\section{9 | Mirror-image morphogenesis in SHFM}

It is noteworthy that the left-right abnormalities of hands and feet in patients affected with SHFM are almost always asymmetric. Although left-right asymmetry of appendages is set very early in development, very little is known about mechanisms that would be susceptible to the asymmetric genesis of abnormalities in processes that occur later in limb development.

Bilaterally, symmetrical organisms exhibit mirror-image morphogenesis of many paired structures, such as limbs, eyebrows, palpebral fissures, nose, and teeth. Over the course of evolution, it appears to provide mechanical advantage for the limbs. It provides balance for the feet and hands and a greater ability to approach things from opposite angles. The embryological basis for bilateral symmetry occurs very early in the embryo with the establishment of the primitive streak, which allows recognition of both the craniocaudal (anteroposterior) and right-left axes. SHFM is evidently a malformation affecting predominantly the anteroposterior axis of the limbs. Sequential expression of Hox genes along the central craniocaudal axis provides the basis for molecular events leading to the development of the left and right fore- and hindlimbs. Until establishment of the anteroposterior axis within the limb field, bilateral symmetry of limbs is not fixed. A century ago, Harrison ${ }^{56}$ and Swett ${ }^{57}$ rotated limb disks or early salamander embryos. If performed early enough, a left limb disk could be converted into a right limb by transplanting it onto the right side of the embryo. Later in development, after the anteroposterior axis was established, the same manipulation resulted in the formation of a left limb, although reversed in orientation. Fixation of the anteroposterior axis occurs very early, before formation of the AER. In birds and mammals, establishment of the ZPA is a concrete indication that the anteroposterior polarity of the limb bud has been fixed.

It is noteworthy that most pathways that lead to SHFM are involved Dlx5 gene, and it is interesting to note that olfactory organs of Dlx5-/- mice are usually asymmetric. ${ }^{58}$ This suggests that Dlx5 may affect the manifestation of mirror-image morphogenesis

\section{4 | CONCLUDING REMARKS}

SHFM is associated with genes involved in AER function or maintenance. It is the consequence of maldevelopment of the central part of the AER, especially as a result of dysregulation of Fgf8. Dysregulation of the p63-Dlx transcriptional pathway appears to affect a centralized network relevant to SHFM. Mutations in WNT10B, FGFR1, or inactivation of Fgf8 disrupt the p63-Dlx-Fgf8 transcriptional network leading to dysregulation of Fgf8 in AER cells, misexpression of genes important for limb development, failure of AER stratification, and subsequently cause SHFM. It is noteworthy that p63-Dlx-Fgf8 transcriptional network is tissue-specific and sensitive to gene dosage, timing, and position. Another pathway leading to SHFM appears to be a Wnt- $\beta$-catenin-Sp8-Dlx-Fgf8 signaling pathway, which does not appear to involve p63. This review shows that a number of molecules are involved in the formation of hands and feet and every molecule seems to be connected. When mutation occurs, the process of limb formation is disrupted like "domino effect," leading to SHFM.

\section{ACKNOWLEDGEMENTS}

We thank our patients and their families for their kind cooperation and for allowing us to use their medical and dental information for the benefit of others. We thank Dr. Chananya Hokierti for Figure 2. This work was supported by The Center of Excellence in Medical Genetics Research, Chiang Mai University; the Thailand Research Fund; The Dental Association of Thailand; and The Faculty of Dentistry, Chiang Mai University. 


\section{Conflict of interest}

All authors declare no conflict of interest.

\section{Editorial Process File}

The Editorial Process File is available in the online version of this article.

\section{ORCID}

Piranit N. Kantaputra (D) https://orcid.org/0000-0001-9841-0881

\section{REFERENCES}

1. Ugur SA, Tolun A. Homozygous WNT10B mutation and complex inheritance in split-hand/foot malformation. Hum Mol Genet. 2008;17: 2644-2653.

2. Lo lacono N, Mantero S, Chiarelli A, et al. Regulation of Dlx5 and Dlx6 gene expression by $p 63$ is involved in EEC and SHFM congenital limb defects. Development. 2008;135:1377-1388.

3. Haro E, Delgado I, Junco M, et al. Sp6 and Sp8 transcription factors control AER formation and dorsal-ventral patterning in limb development. PLoS Genet. 2014;10:e1004468. https://doi.org/10.1371/ journal.pgen.1004468.

4. Petit F, Sears KE, Ahituv N. Limb development: a paradigm of gene regulation. Nature Revs Genet. 2017;18:245-258.

5. van Bokhoven $\mathrm{H}$, Hamel BC, Bamshad M, et al. p63 gene mutations in EEC syndrome, limb-mammary syndrome, and isolated split hand-split foot malformation suggest a genotype-phenotype correlation. Am J Hum Genet. 2001;69:481-492.

6. Gieruszczak-Bialek D, Oldak M, Skorka A, Waligora J, Korniszewski L. Fibular aplasia with ectrodactyly-broadening the clinical spectrum. Eur J Med Genet. 2006;49:83-86.

7. Shamseldin HE, Faden MA, Alashram W, Alkuraya FS. Identification of a novel DLX5 mutation in a family with autosomal recessive split hand and foot malformation. J Med Genet. 2012;49:16-20.

8. Wang $X$, Xin Q, Li L, et al. Exome sequencing reveals a heterozygous DLX5 mutation in a Chinese family with autosomal-dominant split-hand/foot malformation. Eur J Hum Genet. 2014;22:1105-1110.

9. Ullah A, Hammid A, Umair M, Ahmad W. A novel heterozygous intragenic sequence variant in DLX6 probably underlies first case of autosomal dominant split-hand/foot malformation type 1. Mol Syndromol. 2017;8:79-84.

10. Villanueva C, Jacobson-Dickman E, Xu C, et al. Congenital hypogonadotropic hypogonadism with split hand/foot malformation: a clinical entity with a high frequency of FGFR1 mutations. Genet Med. 2015; 17:651-659.

11. Klopocki E, Lohan S, Doelken SC, et al. Duplications of BHLHA9 are associated with ectrodactyly and tibia hemimelia inherited in non-Mendelian fashion. J Med Genet. 2012;49:119-125.

12. Malik S, Percin FE, Bornholdt $D$, et al. Mutations affecting the BHLHA9 DNA-binding domain cause MSSD, mesoaxial synostotic syndactyly with phalangeal reduction, Malik-Percin type. Am J Hum Genet. 2014;95:649-659.

13. Huh JK, Chung MS, Baek GH, Oh JH, Lee YH, Gong HS. Cleft hand in Kabuki make-up syndrome: case report. J Hand Surg Am. 2011;36: 653-657.

14. Conte D, Garaffo G, Lo lacono N, et al. The apical ectodermal ridge of the mouse model of ectrodactyly DIx5;Dlx6-/- shows altered stratification and cell polarity, which are restored by exogenous Wnt5a ligand. Hum Mol Genet. 2016;25:740-754.

15. Umair M, Ullah A, Abbas S, Ahmad F, Basit S, Ahmad W. First direct evidence of involvement of a homozygous loss-of-function variant in the EPS15L1 gene underlying split-hand/split-foot malformation. Clin Genet. 2018;93:699-702.

16. Bakkers J, Hild M, Kramer C, Furutani-Seiki M, Hammerschmidt M. Zebrafish DeltaNp63 is a direct target of Bmp signalling and encodes a transcriptional repressor blocking neural specification in the ventral ectoderm. Dev Cell. 2002;2:617-627.

17. Mills AA, Zheng B, Wang XJ, Vogel H, Roop DR, Bradley A. p63 is a p53 homologue required for limb and epidermal morphogenesis. Nature. 1999;398:708-713.

18. Yang A, Schweitzer R, Sun D, et al. p63 is essential for regenerative proliferation in limb, craniofacial and epithelial development. Nature. 1999;398:714-718.

19. Kjaer KW, Hansen L, Schwabe GC, et al. Distinct $C D H 3$ mutations cause ectodermal dysplasia, ectrodactyly, macular dystrophy (EEM syndrome). J Med Genet. 2005;42:292-298.

20. Ullah A, Ullah MF, Khalid ZM, Ahmad W. Novel heterozygous frameshift mutation in distal-less homeobox 5 underlies isolated split hand/foot malformation type 1. Pediatr Int. 2016;58:1348-1350.

21. Merlo GR, Paleari L, Mantero S, et al. Mouse model of split hand/foot malformation type I. Genesis. 2002;33:97-101.

22. Robledo RF, Rajan L, Li X, Lufkin T. The Dlx5 and Dlx6 homeobox genes are essential for craniofacial, axial, and appendicular skeletal development. Genes Dev. 2002;16:1089-1101.

23. Panganiban G, Rubenstein JL. Developmental functions of the distal-less/dlx homeobox genes. Development. 2002;129:4371-4386.

24. Restelli M, Lopardo T, Lo lacono N, et al. DLX5, FGF8 and the Pin1 isomerase control $\Delta \mathrm{Np} 63 \alpha$ protein stability during limb development: a regulatory loop at the basis of the SHFM and EEC congenital malformations. Hum Mol Genet. 2014;23:3830-3842.

25. Restelli M, Molinari E, Marinari B, et al. FGF8, c-Abl and p300 participate in a pathway that controls stability and function of the $\Delta \mathrm{Np} 63 \alpha$ protein. Hum Mol Genet. 2015;24:4185-4197.

26. Crackower MA, Motoyama J, Tsui LC. Defect in the maintenance of the apical ectodermal ridge in the Dactylaplasia mouse. Dev Biol. 1998;201:78-89.

27. Paina $S$, Garzotto D, DeMarchis $S$, et al. Wnt5a is a transcriptional target of dlx homeogenes and promotes differentiation of interneuron progenitors in vitro and in vivo. J Neurosci. 2011;31:2675-2687.

28. Geetha-Loganathan $P$, Nimmagadda S, Scaal M. Wnt signaling in limb organogenesis. Organogenesis. 2008;4:109-115.

29. Barrow JR, Thomas KR, Boussadia-Zahui $O$, et al. Ectodermal Wnt3/beta-catenin signalling is required for the establishment and maintenance of the apical ectodermal ridge. Genes Dev. 2003;17:394-409.

30. Kawakami Y, Capdevila J, Büscher D, Itoh T, Rodríguez Esteban C, Izpisúa Belmonte JC. WNT signals control FGF-dependent limb initiation and AER induction in the chick embryo. Cell. 2001;104:891-900.

31. Kantaputra PN, Hutsadaloi A, Kaewgahya M, et al. WNT10B mutations associated with isolated dental anomalies. Clin Genet. 2018;93: 992-999.

32. Witte F, Dokas J, Neuendorf F, Mundlos S, Stricker S. Comprehensive expression analysis of all Wnt genes and their major secreted antagonists during mouse limb development and cartilage differentiation. Gene Expr Patterns. 2009;9:215-223.

33. Gros J, Hu JK-H, Vinegoni C, Feruglio PF, Weissleder R, Tabin CJ. WNT5A/JNK and FGF/MAPK pathways regulate the cellular events shaping the vertebrate limb bud. Curr Biol. 2010;20:1993-2002.

34. Ho HY, Susman MW, Bikoff JB, et al. Wnt5a-Ror-Dishevelled signalling constitutes a core developmental pathway that controls tissue morphogenesis. Proc Natl Acad Sci U S A. 2012;109:4044-4051.

35. Yamaguchi TP, Bradley A, McMahon AP, Jones S. A. Wnt5a pathway underlies outgrowth of multiple structures in the vertebrate embryo. Development. 1999;126:1211-1223.

36. Kengaku M, Capdevila J, Rodriguez-Esteban C, et al. Distinct WNT pathways regulating AER formation and dorsoventral polarity in the chick limb bud. Science. 1998;280:1274-1277.

37. Talamillo A, Delgado I, Nakamura T, et al. Role of Epiprofin, a zinc-finger transcription factor, in limb development. Dev Biol. 2010; 337:363-374.

38. Bell SM, Schreiner CM, Waclaw RR, Campbell K, Potter SS, Scott WJ. Sp8 is crucial for limb outgrowth and neuropore closure. Proc Natl Acad Sci U S A. 2003;100:12195-12200.

39. Choi KS, Lee C, Maatouk DM, Harfe BD. Bmp2, Bmp4 and Bmp7 are co-required in the mouse AER for normal digit patterning but not limb outgrowth. PLoS One. 2012;7:e37826. https://doi.org/10.1371/ journal.pone.0037826. 
40. Schatz O, Langer E, Ben-Arie N. Gene dosage of the transcription factor Fingerin (bHLHA9) affects digit development and links syndactyly to ectrodactyly. Hum Mol Genet. 2014;23:5394-5401.

41. Kataoka K, Matsushima T, Ito $\mathrm{Y}$, Sato T, Yokoyama S, Asahara H. Bhlha9 regulates apical ectodermal ridge formation during limb development. J Bone Miner Metab. 2018;36:64-72.

42. Spielmann $\mathrm{M}$, Kakar $\mathrm{N}$, Tayebi $\mathrm{N}$, et al. Exome sequencing and CRISPR/Cas genome editing identify mutations of ZAK as a cause of limb defects in humans and mice. Genome Res. 2016;26:183-191.

43. Saito D, Yonei-Tamura S, Kano K, Ide H, Tamura K. Specification and determination of limb identity: evidence for inhibitory regulation of Tbx gene expression. Development. 2002;129:211-220.

44. Lewandoski M, Sun X, Martin GR. Fgf8 signalling from the AER is essential for normal limb development. Nat Genet. 2000;26:460-463.

45. Guerrini L, Costanzo A, Merlo GR. A symphony of regulations centered on p63 to control development of ectoderm-derived structures. J Biomed Biotechnol. 2011;864904:1-13. https://doi.org/10.1155/201 $1 / 864904$.

46. Zeller R, López-Ríos J, Zuniga A. Vertebrate limb bud development: moving towards integrative analysis of organogenesis. Nat Rev Genet. 2009;10:845-858.

47. Gañan Y, Macias D, Basco RD, Merino R, Hurle JM. Morphological diversity of the avian foot is related with the pattern of msx gene expression in the developing autopod. Dev Biol. 1998;196:33-41.

48. Kaltcheva MM, Anderson MJ, Harfe BD, Lewandoski M. BMPs are direct triggers of interdigital programmed cell death. Dev Biol. 2016; 411:266-276

49. Khan A, Wang R, Han S, Ahmad W, Zhang X. A novel homozygous missense mutation in BHLHA9 causes mesoaxial synostotic syndactyly with phalangeal reduction in a Pakistani family. Hum Genome Var. 2017, 2017;4:17054. https://doi.org/10.1038/hgv.2017.54. eCollection.
50. Tickle C. Making digit patterns in the vertebrate limb. Nat Rev Mol Cell Biol. 2006;7:45-53.

51. Hiscock TW, Tschopp P, Tabin CJ. On the formation of digits and joints during limb development. Devel Cell. 2017;41:459-465.

52. Harfe BD, Scherz PJ, Nissim S, Tian H, McMahon AP, Tabin CJ. Evidence for an expansion-based temporal Shh gradient in specifying vertebrate digit identities. Cell. 2004;118:517-528.

53. Litingtung Y, Dahn RD, Li Y, Fallon JF, Chiang C. Shh and Gli3 are dispensable for limb skeleton formation but regulate digit number and identity. Nature. 2002;418:979-983.

54. Conte D, Guerrini L, Merlo GR. Novel cellular and molecular interactions during limb development, revealed from studies on the split hand foot congenital malformation. In: Wu B, ed. New Discoveries in Embryology. INTECH; London, United Kingdom 2015:125-167.

55. Suzuki T, Hasso SM, Fallon JF. Unique SMAD $1 / 5 / 8$ activity at the phalanx-forming region determines digit identity. Proc Natl Acad Sci U S A. 2008;105:4185-4190.

56. Harrison RG. On relations of symmetry in transplanted limbs. J Exp Zool. 1921;32:1-136.

57. Swett FH. Determination of limb-axes. Q Rev Biol. 1937;2:322-339.

58. Long JE, Garel S, Depew MJ, Tobet S, Rubenstein JL. DLX5 regulates development of peripheral and central components of the olfactory system. J Neurosci. 2003;23:568-578.

How to cite this article: Kantaputra PN, Carlson BM. Genetic regulatory pathways of split-hand/foot malformation. Clin Genet. 2019;95:132-139. https://doi.org/10.1111/cge.13434 